\title{
Clinical Observation on Effect of Wrist-Ankle Acupuncture Combined with Ashi Acupuncture Point on Muscle Strain of Aerobics Athletes
}

\author{
AIHUA YANG AND L. LI ${ }^{1 *}$ \\ School of Physical Education, Changsha University of Science \& Technology, Changsha 410114, ${ }^{1}$ School of Physical Education, \\ Hunan University of Science and Technology, Changsha 412201, China
}

Yang et al.: Effect of Wrist-Ankle Acupuncture Combined with Ashi Acupuncture Point on Muscle Strain This paper aims to evaluate the clinical curative effect of wrist-ankle acupuncture combined with Ashi acupuncture point for the treatment of muscular strain in aerobics athletes and to provide scientific reference for future clinical treatment. 190 aerobics athletes with muscle strain treated in our hospital from March 2014 to March 2016 were randomly divided into two groups according to the random double blind method. The 95 patients in the observation group were treated with wrist-ankle acupuncture combined with Ashi acupuncture point, while the patients in the control group received body acupuncture alone. The improvement of muscle strain was compared between two groups and effective rate of treatment was evaluated. The average time of improvement in muscle strain was $(3.75 \pm 1.23) \mathrm{d}$ for the observation group and $(6.17 \pm 1.42) \mathrm{d}$ for the control group. Comparison between the two groups $(\mathrm{p}<0.05)$ was with significant statistical significance; the overall effective rate was $98.95 \%(94 / 95)$ in the observation group and $90.53 \%$ $(86 / 95)$ in the control group. There was significant difference between the two groups $(\mathbf{p}<0.05)$. However, the cure rate was $81.05 \%$ (77/95) for the observation group, but $60 \%(57 / 95)$ for the control group. Comparison between the two groups $(p<0.05)$ was with significant statistical significance. Wrist-ankle acupuncture combined with Ashi acupuncture point can effectively shorten the wound healing time and improve the cure rate of muscle strain, which has high clinical application and popularization value.

Key words: Wrist-ankle acupuncture, Ashi acupuncture point, muscle strain in aerobics, clinical curative effect

Aerobics is a new sport in recent years, in which the athletes complete a variety of actions with rhythmic music accompaniment in order to achieve the purpose of fitness, body building and health. Aerobics training objectives and action requirements are different, which are divided into public fitness and athletic body building. Public fitness gymnastics has lower requirement for action, smaller exercise intensity and load. However, athletic gymnastics requires a high degree of movement, with smaller exercise intensity and load and anaerobic functional metabolism as the main. But both need basic quality such as flexibility, sensitivity, strength as a guarantee and so they are more prone to sports injuries. The statistical results show that injury rate of gymnastics is about $58.72 \%$ due to poor technical level, excessive physical activity and environmental factors, while muscle strain is the most common injury except for joint sprain and ligament injury.

Muscle strain (fig. 1-3) is a muscle injury caused by sharp contraction or excessive traction in the movement. It can be easily caused in trample, chin up and sit ups exercises ${ }^{[1]}$. After muscle strain, the strain parts suffer sharp pain, one can touch block like lumps with significant pain due to muscle tension. Due to local swelling or subcutaneous bleeding, activity will be significantly limited. In sports, due to improper preparation, physiological function of a part of muscle has not yet reached the required state to adapt to the movement; training level is not enough, muscle flexibility and strength is poor; fatigue or excessive load results in muscle function decline and weakening and reduced coordination; wrong technical action or no concentrated attention during exercise, overexertion or

*Address for correspondence

E-mail: yuqf1818@163.com 
roughness; too low humidity and too much humidity, poor quality of the site or equipment can lead to muscle strain.

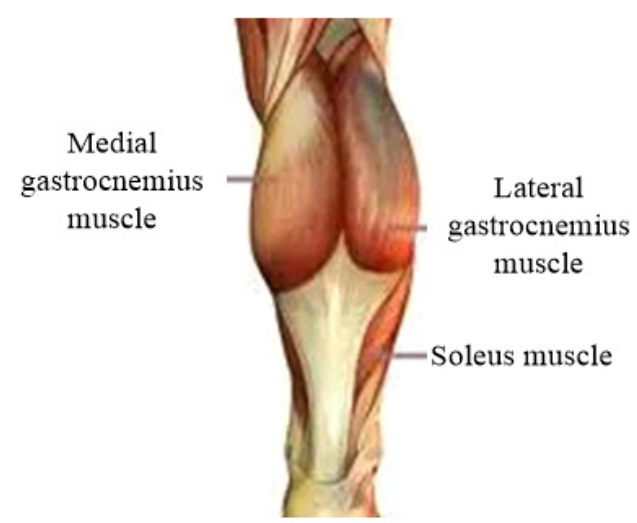

Fig. 1: Calf muscle strain

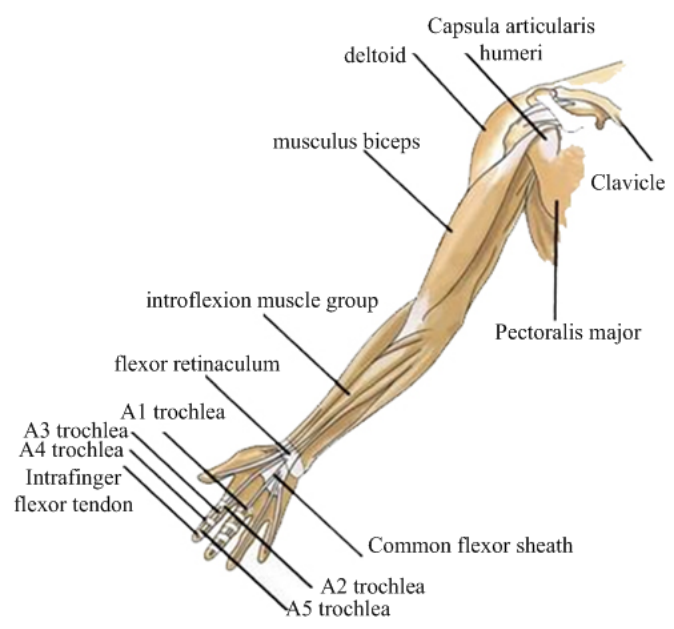

Fig. 2: Arm muscle strain

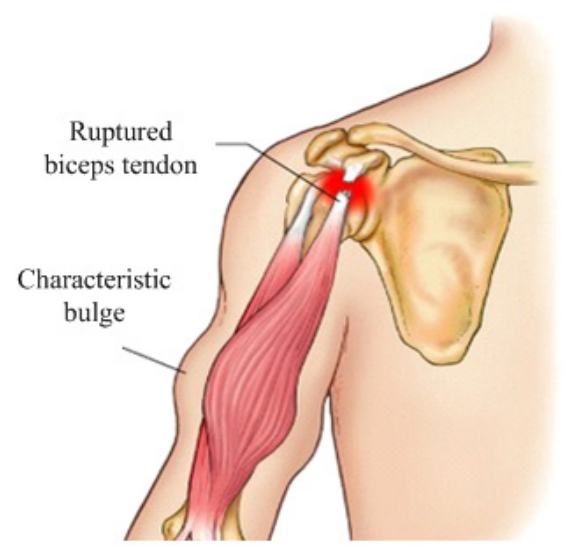

Fig. 3: Shoulder muscle strain

Clinical treatment of muscle strain usually follows basic principles of relieving pain, preventing stasis, promoting menstruation, laying bones, expelling wind and removing cold. Ashi acupuncture point therapy is a very common treatment method. But according to research results of Zhou Bencheng, Shenjian, cure rate of Ashi acupuncture point was only $61.3 \%$ for muscle strain in aerobics, which cannot meet the requirement of restoring patient's physical activity ability as soon as possible $\mathrm{e}^{[2]}$. Therefore, in our study, our hospital tried to combine Ashi acupuncture point therapy with wristankle acupuncture therapy, in order to further improve the cure rate.

\section{MATERIALS AND METHODS}

\section{General Information}

The subjects of this study were 190 cases of aerobics athletes with muscle strain treated in our hospital from March 2014 to March 2016, who was randomly divided into two groups with double blind method, each group with 95 cases. The observation group consisted of 42 males and 53 females aged between 12 and $26 \mathrm{y}$. The course of the disease ranged from 0.5 to $13 \mathrm{~d}$. The control group consisted of 44 males and 51 females aged from 11 to $27 \mathrm{y}$. The course of the disease ranged from 1 to $15 \mathrm{~d}$. The two groups were in line with the clinical diagnosis of muscle strain (fig. 4 and fig. 5). The injury sites included 10 cases of back muscle, 18 cases of shoulder muscle, 42 cases of gastrocnemius, 27 cases of sartorius muscle, 34 cases of popliteus muscle and 59 cases of quadriceps femoris, which were evenly distributed among the two groups (Table 1).

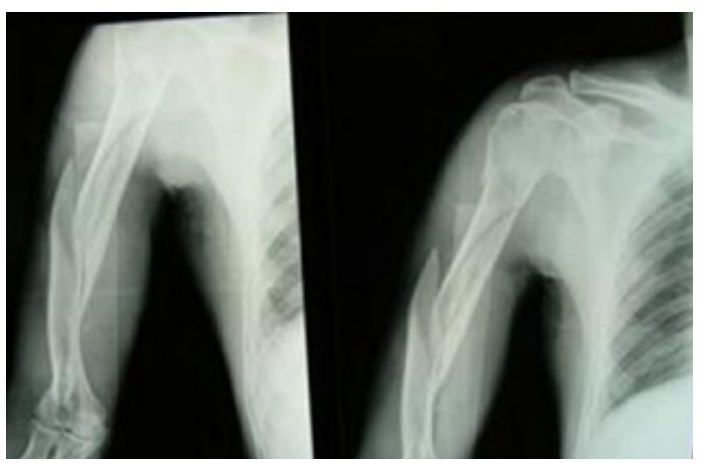

Fig. 4: X-ray film of 1 case of shoulder muscle strain

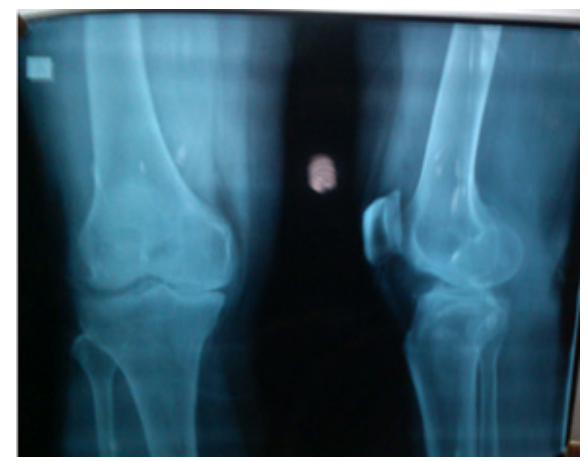

Fig. 5: X-ray film of 1 case of calf muscle strain 
www.ijpsonline.com

TABLE 1: COMPARISON OF GENERAL TREATMENT OF THE TWO GROUPS N=95

\begin{tabular}{lccc}
\hline Group & Gender composition & Average age/y & Average duration/d \\
\hline Observation group & Male 42/female 53 & $18.5 \pm 2.4$ & $6.23 \pm 1.12$ \\
Control group & Male 44/female 51 & $18.7 \pm 2.2$ & $6.41 \pm 1.08$ \\
$\mathrm{p}$ & $>0.05$ & $>0.05$ & $>0.05$ \\
\hline
\end{tabular}

Inclusion and exclusion criteria ${ }^{[3]}$ : Have a history of significant sports injury; partial muscle pain, tenderness, swelling and subcutaneous bleeding, resistance pain $(+)$; some patients may have palpable block like mass, with accompanying tearing of muscle fascia; patients with complete rupture of muscle fascia and presence of other types of injury were excluded; all patients were willing to participate in treatment research and signed informed consent and the relevant information was approved by the Ethics Committee.

\section{Therapeutic method}

The control group was treated with body acupuncture only. For acupuncture of strain site, neck and shoulder include Yanglao, Jian Jing, Dazhui, neck and back include Tianzong, Xuanzhong, anterior part of chest includes Neiguan through Wai kuan, lateral thorax part includes Taichong, center of the waist includes Renzhong, both sides include Yanglao, lateral thigh includes Zusanli, Yanglingquan, posterior leg includes Feiyang. For a large area of strain, add 4-6 needles at upper and lower, left and right edge of the pain zone ${ }^{[4]}$. Lift and pull to produce a sense of needle, manipulate needle once every $5 \mathrm{~min}$, each treatment lasts $30 \mathrm{~min}$, once a day. Every $3 \mathrm{~d}$ represent completion of a course of treatment.

The observation group was treated with wrist-ankle acupuncture combined with Ashi acupuncture point. First, find the most painful point in the affected side of the patient, that is, Ashi point. Conduct routine disinfection for local skin and choose appropriate needle according to the injured muscle depth, generally $50-75 \mathrm{~mm}$ in length. Pierce the pain center, lift, thrust, swirl and rotate for $1 \mathrm{~min}$ after arrival of $\mathrm{qi}^{\mathrm{i}}{ }^{[\mathrm{j}}$. And then select acupoint by referring to the wrist-ankle acupoint selection method based on the injury site, take upper 4, 5 acupuncture of the affected side of the shoulder; take upper 5 acupuncture of the affected side of the elbow; take upper 5 acupuncture of the affected side of the wrist; take double lower 5,6 acupuncture of the waist; take lower 3, 4 acupuncture of the affected side of the knee; take lower 5 acupuncture of the affected side of the lateral malleolus; take lower 2 acupuncture of the affected side of the malleolus medialis. Before treatment, conduct routine skin disinfection and then select No. 30-32, 30-40 mm long stainless steel needle ${ }^{[6]}$, make a $30^{\circ}$ angle between the needle body and the skin surface, slightly spin the needle and quickly penetrate it into the skin and then lay the needle flat, point the needle to the lesion, gently push the needle along the skin about $2.5 \mathrm{~cm}$ until needle protrusion can be seen on the body surface. When pushing the needle, the doctor should not have a sense of resistance in the fingers, the patient should not have acid, hemp, swelling or heavy pain feeling, otherwise, withdraw the needle to repenetrate it. Retain the needle for $30 \mathrm{~min}^{[7]}$, generally no hand manipulation of needle is needed during needle retention. For some patients, to enhance the analgesic effect, scraping needle approach can be used. Treat once daily and for $3 \mathrm{~d}$ represent the course of treatment.

\section{Clinical observation indicators}

The time of improvement in muscle strain in the two groups was recorded in detail and the effect was further determined according to the symptoms, palpation examination, functional recovery and the actual situation of the patients ${ }^{[8,9]}$. Invalid: After 15 times of treatment or more, there was no significant relief of symptoms, patient could not carry out daily training. Improved: After treatment, pain, swelling, tenderness was relieved, but the functional recovery was poor, patient could not engage in training of moderate or above intensity. Markedly: After treatment, pain was significantly reduced, swelling and tenderness subsided, function basically recovered, there was no or only mild symptoms during rest, symptoms slightly heightened after training, patient could not engage in high intensity training in daily training, but moderate intensity training was allowed ${ }^{[10]}$. Cured: After treatment, pain and local tenderness disappeared, swelling subsided, function recovered, routine training was as usual and patient could carry out normal moderate, high intensity training.

Cure rate $=$ cured/total number of people $\times 100 \%$ Effective rate $=($ improved + markedly + cured $) /$ total number of people $\times 100$ 


\section{Statistical methods}

In this study, the clinical effects of wrist-ankle acupuncture combined with Ashi acupuncture point in treatment of muscle strain of aerobics athletes were analyzed. The data was analyzed and processed with SPSS 19.0 statistical software. The count data were expressed as (n, \%) and tested by chi square, while the measurement data was expressed as $(\overline{\mathrm{x}} \pm \mathrm{s})$ and tested by $t$. The difference was statistically significant only if $\mathrm{p}<0.05$ was satisfied.

\section{RESULTS AND DISCUSSION}

The average time of improvement in muscle strain was
$(3.75 \pm 1.23) \mathrm{d}$ for the observation group and $(6.17 \pm 1.42)$ $\mathrm{d}$ for the control group. The difference between the two groups $(\mathrm{t}=12.555, \mathrm{p}=0.000<0.05)$ was with significant statistical differences (Table 2).

The total effective rate was $98.95 \%(94 / 95)$ in the observation group and $90.53 \%(86 / 95)$ in the control group, the difference was statistically significant $(\mathrm{p}<0.05)^{[11]}$. However, the cure rate was $81.05 \%(77 / 95)$ for the observation group and only $60 \%$ (57/95) for the control group, the comparison between the two groups $(\mathrm{p}<0.05)$ was with significant statistical significance (Table 3).

TABLE 2: COMPARISON OF TIME OF IMPROVEMENT IN MUSCLE STRAIN OF TWO GROUPS OF PATIENTS WITH EFFECTIVE TREATMENT (N, \%)

\begin{tabular}{lccc}
\hline Group & $\leq 3 \mathrm{~d}$ & $4-6 \mathrm{~d}$ & $>6 \mathrm{~d}$ \\
\hline Observation group $(\mathrm{n}=94)$ & $68(72.34)$ & $21(22.34)$ & $5(5.32)$ \\
Control group $(\mathrm{n}=86)$ & $42(48.84)$ & $27(31.40)$ & $17(19.76)$ \\
\hline
\end{tabular}

TABLE 3: COMPARISON OF TREATMENT EFFECT OF THE TWO GROUPS [(N, \%), N=95]

\begin{tabular}{lcccccc}
\hline Group & Invalid & Improved & Markedly & Cured & Effective rate & Cure rate \\
\hline $\begin{array}{l}\text { Observation } \\
\text { group }\end{array}$ & 1 & 6 & 11 & 77 & $98.95 \%$ & $81.05 \%$ \\
Control group & 9 & 11 & 18 & 57 & $90.53 \%$ & $60 \%$ \\
$x^{2}$ & & & & & 6.756 & 10.128 \\
$\mathrm{p}$ & & & & & 0.009 & 0.001 \\
\hline
\end{tabular}

From the perspective of traditional Chinese medicine, muscle strain belongs to "injured tendon", a symptom of damaged muscle meridian and collaterals. The main pathogenesis is adverse muscle meridian, blood barrier. For treatment, it is better to activate blood and remove stasis, promote qi circulation to relieve pain. Ashi point is the main point of pathological response. Based on principle of adjacent acupoint selection, to stimulate the point, we can activate blood, remove stasis and clear the meridians, so it is more commonly used ${ }^{[12]}$.

Wrist-ankle acupoint is a subcutaneous needle retention method, which works mainly through stimulation of twelve skin areas which are corresponding parts of twelve meridians function reflected in the body surface, also area with collaterals distribution. The stimulation of twelve skin areas can regulate twelve meridians, dredge the meridians, regulate qi and blood and stimulate channel qi running of the skin. Wrist-ankle acupuncture, through the neural and humoral regulation and the combined effect of a variety of automatic control systems of the living organism, can mitigate spasm of the lesion site, improve local blood circulation and nutrition of injured limb and promote absorption of edema and inflammation to achieve analgesic effect. As this method features less acupoint selection than pure acupuncture, slight pain and does not need soreness which is required by general acupuncture, it enjoys greater advantages in clinics. The study results showed clear advantage in treatment effect of observation group compared with the control group. Also, time of improvement in muscle strain was significantly shorter than that of the control group, which further demonstrated effectiveness of Ashi point combined with wrist-ankle acupuncture.

In summary, wrist-ankle acupuncture combined with Ashi point for muscle strain in aerobics can effectively improve the treatment efficiency, which enjoys greater advantages in efficiency and cure rate compared with simple body acupuncture treatment. Hence, it is recommended that this combination therapy can be widely used in clinics. 


\section{Conflicts of Interest:}

The authors declared no conflict of interest.

\section{REFERENCES}

1. Zhou BC, Shen J. Clinical observation on effect of wrist-ankle acupuncture combined with Ashi acupuncture point on muscle strain of aerobics athletes. Chin J Sports Med 2012;31(9):826-7.

2. Wang XC. Clinical observation of Ashi Point with auxiliary low-frequency electro-acupuncture drug in treatment of cervicogenic headache. Chin J Pain Med 2014;20(7):526-8.

3. Wu F, Kang M, Xiong P. Clinical randomized controlled observation of Ashi Point combined with moxibustion of heat sensibilizing state of acupoints in treatment of back myofascial pain syndrome. Acupuncture Res 2011;36(2):116-20.

4. Xiao M, Wang Q, Ren W, Zhang Z, Wu X, Wang Z, et al. Impact of prediabetes on poststroke depression in Chinese patients with acute ischemic stroke. Int J Geriatr Psychiatry 2018;33(7):956-63.

5. Chen RN, Chen YB. Clinical observation on therapeutic effect and instant analgestic effect of inhibitory needling at Ashi point as major point for treatment of piriformis syndrome. Chin Acupuncture Moxibustion 2009;9(7):550-2.

6. Duan H, Huang F. Clinical observation of 40 cases of neck and shoulder pain treated with acute acupuncture of houxi point combined with Ashi acupuncture point. World Health Dig 2013;12(10):362.

7. De Souza Mitidieri AM, Baltazar MC, Pht AP, Gurian MB, Poli-Neto OB, Candido-Dos-Reis FJ, et al. Ashi acupuncture versus local anesthetic trigger point injections in the treatment of abdominal myofascial pain syndrome: A randomized clinical trial. Pain Physician 2020;23:507-17.
8. Hsu WC, Guo SE, Chang CH. Back massage intervention for improving health and sleep quality among intensive care unit patients. Nurs Crit Care 2019;24(5):313-9.

9. Dong B, Lin L, Chen Q, Qi Y, Wang F, Qian K, et al. Wristankle acupuncture has a positive effect on cancer pain: a metaanalysis. BMC Complement Med Ther 2021;21(1):1-10.

10. Liang S, Zhang G, Li J, Zhong L, Zhang C. Wrist ankle acupuncture in the treatment of acute lumbar sprain: A protocol for systematic review and meta-analysis. Medicine 2020;99(49):e23420.

11. Shi P, Du J, Fang F, Yu H, Liu J. Design and Implementation of an Intelligent Analgesic Bracelet Based on Wristankle Acupuncture. IEEE Trans Biomed Circuits Syst 2020;14(6):1431-40.

12. You Y, Zhang T, Shu S, Qian X, Zhou S, Yao F. Wrist-ankle acupuncture and fluoxetine in the treatment of post-stroke depression: a randomized controlled clinical trial. J Tradit Chin Med 2020;40(3):455-60.

This is an open access article distributed under the terms of the Creative Commons Attribution-NonCommercial-ShareAlike 3.0 License, which allows others to remix, tweak, and build upon the work non-commercially, as long as the author is credited and the new creations are licensed under the identical terms

This article was originally published in a special issue,
"Trends in therapeutic Management of Various Clinical
Conditions II" Indian J Pharm Sci 2021:83(2)Spl issue;131-135

